\title{
A case report of atlanto-axial instability in a Down Syndrome patient
}

\author{
Mubashir Alavi Jusabani ${ }^{1}$ Sakina Mehboob Rashid $\mathbb{D}^{2} \cdot$ Honest Herman Massawe $\cdot$ William Patrick Howlett ${ }^{3}$. \\ Marieke Cornelia Johanna Dekker ${ }^{4,5}$
}

Received: 3 September 2018 / Revised: 7 November 2018 / Accepted: 12 November 2018

(C) International Spinal Cord Society 2018

\begin{abstract}
Introduction Down Syndrome (DS) - also referred to as Trisomy 21-is associated with multiple deformities affecting various systems of the body. Joint laxity resulting in unstable atlanto-occipital and atlanto-axial joints—with an increased susceptibility to fatal cord injuries - is a recognized phenomenon in patients with DS.

Case presentation We report on a 30-year-old man with DS from Tanzania with no previous neck complaints, who presented with a 5-week progressive history of paraparesis that evolved into a quadriparesis. During his initial presentation, the patient reported that 5 months ago he had been involved in a headlock during a playground encounter.

Discussion Primary prevention of the traumatic spinal cord injuries is an important public health measure and a heightened emphasis should be placed to prevent such injuries in high risk populations-including DS patients.
\end{abstract}

\section{Introduction}

With an estimated incidence of 1 in 1000, Down Syndrome (DS) is one of the most extensively studied chromosomal disorders that is compatible with life [1].

Studies of the musculoskeletal system in DS patients have demonstrated reduced bone mineral density and ligament laxity. Decreases in overall osteoblast activity and lower lifetime peak bone mass values have been linked to the low bone mineral densities found in patients with DS [2]. Additionally, the connective tissue disorder associated with DS results in ligament laxity, which is linked to joint hyper-flexibility and concomitant instability [3, 4]. Moreover, central hypotonia in DS is one of the neurological

Mubashir Alavi Jusabani

mubashirj92@gmail.com

1 Department of Orthopedics and Traumatology, Kilimanjaro Christian Medical Centre, Moshi, Tanzania

2 Kilimanjaro Christian Medical Centre, Moshi, Tanzania

3 Department of Internal Medicine, Kilimanjaro Christian Medical Centre, Moshi, Tanzania

4 Department of Pediatrics and Child Health, Kilimanjaro Christian Medical Centre, Moshi, Tanzania

5 Department of Neurology, Radboudumc Nijmegen, Nijmegen, Netherlands characteristics that can be recognized from the neonatal period onwards [5].

Ligament laxity has been linked with atlanto-axial instability (AAI). This is a well-described developmental anomaly in DS patients, which is defined as an increased ( $>3$ millimetres in adults) distance between the posterior aspect of the frontal arch of $\mathrm{C} 1$ and the anterior aspect of the dens of $\mathrm{C} 2$ when measured on radiographs taken in the neutral positon [6]. El-Khouri's series of 80 DS patients were assessed for the presence of AAI and 11 (13.7\%) individuals had radiographic evidence of the condition, while only one (1.3\%) patient had symptoms of cervical instability [7]. The reported incidence of AAI in individuals with DS is recorded as between 6.8 and $27.0 \%$ across various reports [8].

In view of the cervical spine in DS, the sum effect of these factors is decreased stability, with potentially fatal outcomes, following injury due to the proximity of brainstem structures, which centrally control the cardiorespiratory drive as well as the level of the spinal cord (C3-C5) innervating the primary muscle of respiration, the diaphragm.

\section{Statement of ethics}

This clinical case study was approved of by the Medical Ethics Committee of Kilimanjaro Christian Medical Centre, Moshi, United Republic of Tanzania, in the framework of a study on neurological disorders in KCMC. Informed consent was obtained from the patient. 


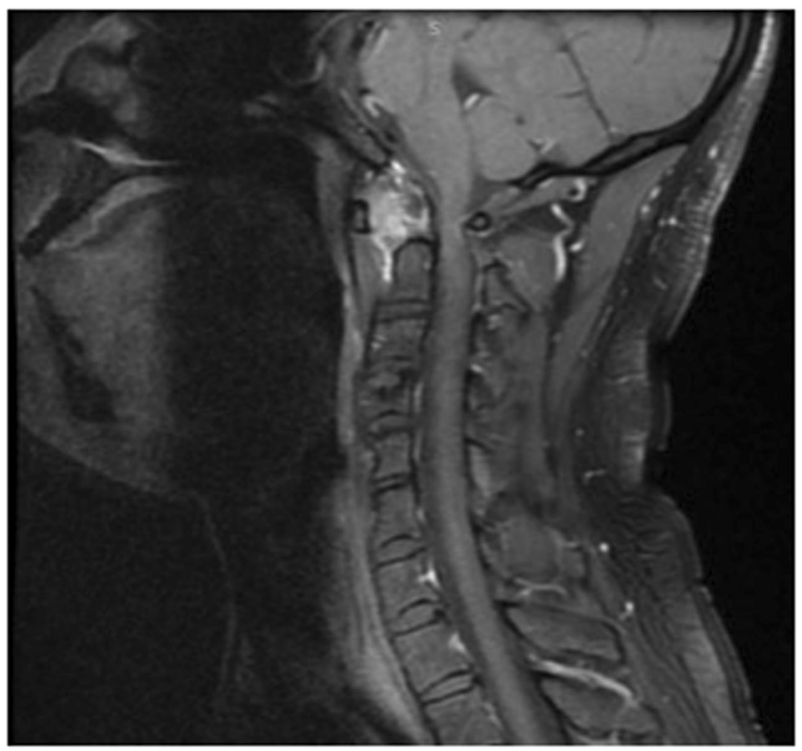

Fig. 1 A sagittal T1-weighted MRI section demonstrating anterior displacement of $\mathrm{C} 1$ over $\mathrm{C} 2$ resulting in compression of the cervical spinal cord

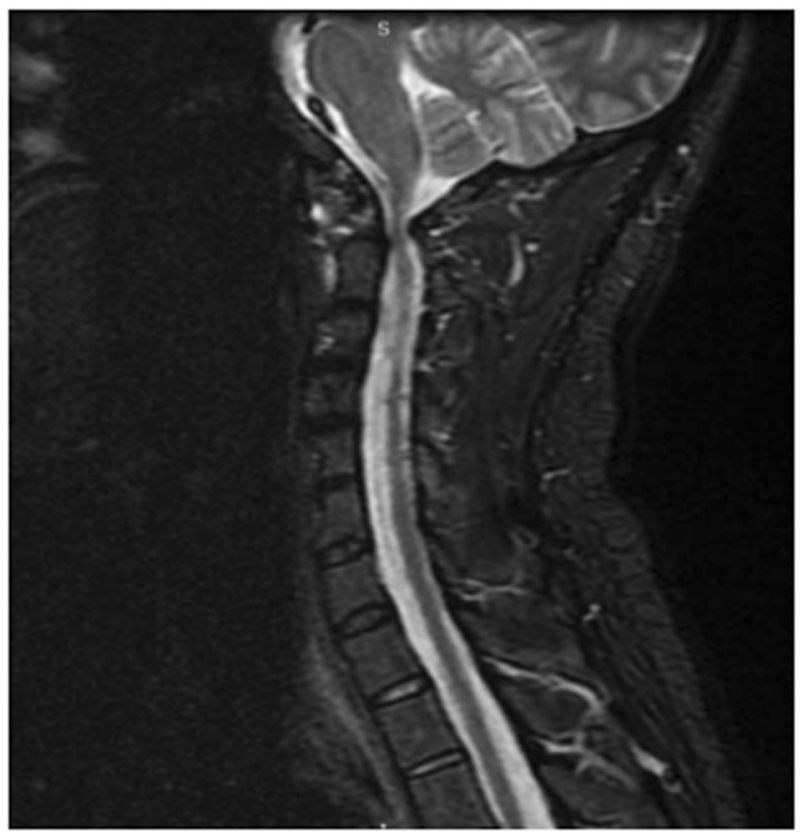

Fig. 2 A sagittal T2-weighted MRI section demonstrating compression of the spinal cord at the level of the $\mathrm{C} 1-\mathrm{C} 2$ vertebrae

\section{Case presentation}

\section{History}

A 30-year-old Tanzanian man with previously undiagnosed DS presented with a 5-week history of neck pain radiating to the occiput, along with lower limb weakness 2 weeks following the onset of the pain. He also reported episodes of dizziness, numbness on the digits of the right upper limb and excessive sweating. Bowel and bladder control was not affected. The patient required support for mobility and was otherwise restricted to a wheelchair. Five months prior to the onset of the complaints, the patient was involved in a headlock during a friendly encounter with his playmates, and no other possibly precipitating factors could be recalled.

On examination, the patient had typical features of DS consisting of epicanthal folds, flat midface, large protruding tongue, brachycephaly, short puffy fingers and a sandal gap between the first and second toe. No heart murmurs were present. He had a normal level of consciousness, was quadriparetic and seated upright on a wheelchair with a neck collar. Cranial nerve examination demonstrated ptosis of the right eye with increased sweating on the right side of the face. The pupils were, however, equal in size, symmetrical and reacted to light. Upon examination of the limbs, no other structural abnormalities were appreciated; tone was generally increased and a greater degree of loss of motor strength was noted in the upper limbs. There was a more profound loss of power in the right upper limb (Proximal: 3/ 5, Distal: 0-1/5) compared to the left upper limb (proximal: 3-4/5, distal: 3-4/5). Motor strength in the lower limbs was bilaterally $4 / 5$, both in proximal and distal muscle groups. Brisk reflexes were appreciated in the upper and lower limbs and clonus observed at the left ankle. Apart from reported paraesthesia in the digits of the right upper limb, the sensory examination was normal (see Table 1).

\section{Diagnosis}

The working diagnosis was DS with spinal cord compression at the level of C1-C2 secondary to atlanto-axial dislocation and occipital nerve compression causing occipital pain. The patient also had a central cord syndrome (CCS) with a greater degree of loss of motor strength noted in the upper limbs compared to the lower limbs.

A magnetic resonance imaging (MRI) study at the level of the cervical spine demonstrated marked anterior atlantoaxial subluxation with a resulting atlanto-axial interval of $7 \mathrm{~mm}$. The resulting severe spinal canal stenosis caused spinal cord compression at the level of $\mathrm{C} 1-\mathrm{C} 2$, with focal cord oedema noted, more marked over the posterior aspect of the cord. The posterior fossa examination was normal and no additional abnormalities were picked following contrast administration (see Figure 1 and 2).

\section{Clinical course-treatment and outcome}

The patient was placed on halter traction for 6 weeks in the flexion position with weights of $4 \mathrm{~kg}$. Serial neurological 
Table 1 The motor neurological examination of the patient

\begin{tabular}{|c|c|c|c|c|}
\hline \multirow[t]{2}{*}{ Examination } & \multicolumn{2}{|l|}{ Right } & \multicolumn{2}{|l|}{ Left } \\
\hline & Upper Limb & Lower Limb & Upper Limb & Lower Limb \\
\hline Inspection & Normal & Normal & Normal & Normal \\
\hline Tone & Increased & Increased & Increased & Increased \\
\hline Power & Proximal: $3 / 5$ Distal: $0-1 / 5$ & $\begin{array}{l}\text { Proximal: } 4 / 5 \\
\text { Distal: } 4 / 5\end{array}$ & $\begin{array}{l}\text { Proximal: } 3-4 / 5 \\
\text { Distal: } 3-4 / 5\end{array}$ & $\begin{array}{l}\text { Proximal: } 4 / 5 \\
\text { Distal: } 4 / 5\end{array}$ \\
\hline Reflexes & Increased & Increased & Increased & Increased \\
\hline Ankle clonus & Absent & Present & & \\
\hline Plantar reflex & Equivocal & Equivocal & & \\
\hline
\end{tabular}

Muscle power was graded in accordance to the MRC (Medical Research Council) Muscle Scale [16] examinations demonstrated improved motor function. Upon discharge, he was also provided with a referral letter for the country's national hospital, where the definitive treatment of $\mathrm{C} 1-\mathrm{C} 2$ posterior fusion could be carried out. The patient opted not to undergo surgery as following the 6 weeks on halter traction there was significant return of power to both his upper and lower limbs.

The family preferred to attend physiotherapy and occupational therapy clinics closer to their home due to travel and treatment costs associated with attending the sessions at KCMC. His progress was followed up by the orthopaedics outpatient clinic at our centre.

During a clinic visit 2 months later the patient was able to ambulate with support (a walking frame) and power had improved globally to grade $4 / 5$. He reported no further occipital pain. His bladder and bowel control was still good.

Six months post injury, the patient was able to brush his teeth, move out of bed and feed independently, with improved grip in his right upper limb. However, he still needed assistance with dressing himself and required support while walking with either a walking frame or holding on to a caregiver's arm. Apart from the assistance the patient required to dress himself and to walk, his degree of independence was similar to the pre-injury level.

\section{Discussion}

We present a 30-year-old Tanzanian man with newly diagnosed DS and previously normal neurological motor performance developing quadriparesis.

The patient's involvement in a headlock-resulting in hyperextension of the neck -5 months prior to presentation in clinic was identified as a possible cause of injury. He was likely pre-disposed to developing the condition due to an existing-but previously unidentified-background of atlanto-axial instability, a disorder secondary to joint laxity, which is characteristic of DS.

Mild compression of the lateral cervical spinal cord has been known to cause contralateral hyperhidrosis above the level of the lesion [9]. Iwase et al.'s series of patients presenting with hemifacial hyperhidrosis and MRI scans in each individual demonstrated mild disc herniation causing slight cervical spinal cord compression without intramedullary changes in signal intensity [9].

The patient's weakness progressively worsened over a period of 3 weeks following the onset of symptoms, suggesting that the dislocation of the atlanto-axial joint was unstable and resulted in continuous insult to the spinal cord. The focal cervical cord oedema noted on the MRI, and differing degree of loss of power picked on the upper and lower limb examination, indicates that the descending motor fibres were injured secondary to the dislocation, albeit to differing degrees.

The patient's clinical presentation, which included a greater degree of loss of motor strength in the upper limbs relative to the lower limbs, also led us to diagnose CCS. Patients received at KCMC following a traumatic SCI are generally assessed for injury severity using the International Standards for Neurological Classification of Spinal Cord Injuries (ISNCSCI) after spinal shock has resolved [10]. In this patient's case, although a documentation of motor strength was regularly maintained, we were unable to find a record of a physical examination done in accordance with the ISNCSCI in his medical records.

Symptomatic AAI occurs in 1-2\% of patients with DS and is often manifested by the presence of upper motor neuron signs on examination, an abnormal gait and neck discomfort-specifically pain, torticollis or a limited range of motion [11]. Less commonly observed are sensory deficits and change in sphincter control [11]. Asymptomatic patients with AAI are often identified during screening practices [7].

Although a posterior fusion of $\mathrm{C} 1$ and $\mathrm{C} 2$ is considered the definitive treatment for AAI [12], our patient was initially placed on cervical traction due to the progressive nature of his symptoms. The traction exerts a therapeutic effect by causing lengthening and relaxation of the muscles around the cervical spine, thus allowing reduction into the anatomical position and alleviation of pressure from the 
cord [12]. It is critical to note that while symptom relief from traction can be quite significant, as observed in our patient, it is not a definitive solution for the pre-existing vertebral and ligamentous instability, which initially resulted in spinal cord injury.

While the patient was admitted in the hospital for 6 weeks of skeletal traction, nursing care involved assisted two-hourly turning and daily inspection for the development of bedsores. The patient was also regularly reviewed by a neurologist, orthopaedic surgeon, physiotherapist and occupational therapist, who are part of the multidisciplinary team that attends to all SCI patients in the hospital. While in the ward, there were no secondary complications encountered as are normally seen in immobilized traumatic SCI patients, such as pressure sores or hypostatic pneumonias.

Definitive management involves spine stabilization surgery. The opportunities for these procedures are limited at KCMC due to the lack of necessary equipment for the orthopaedic surgeons, and referral to specialized centres is often a challenge for patients due to the associated expense of travel and treatment.

The patient was placed on cervical traction for a duration of 6 weeks and serial neurological examination demonstrated significant improvement. Upon discharge from the hospital, the patient and relatives were provided with a referral letter to a higher centre of care, where a posterior fusion of $\mathrm{C} 1$ and $\mathrm{C} 2$ could be carried out. Although they were counselled about the nature of the condition, the associated risk of recurrence and the possibility of a fatal outcome, the family opted not to undergo surgery due to the associated risks and simply because the patient experienced significant improvement following traction. Discussions with the patient and his family also involved stressing the need to avoid potentially cervical-injury-causing activities.

A striking feature of this patient's presentation to hospital was the first-time diagnosis of Down Syndrome. Tanzania is a low-income country with citizens having limited access to healthcare, and in a society where traditional medicine is still largely practised it is not uncommon for a spectrum of conditions to go undiagnosed for prolonged periods of time [13].

Wellness checks for new-borns do exist in the form of clinic attendance for routine vaccinations although a majority of the clinics are run by healthcare providers who are only trained to screen for the most basic and immediately life-threatening conditions. Tanzania's doctor to population ratio stands at roughly 1:45,000 [14].

While the phenotype of DS can lead to an early diagnosis at birth, the poor healthcare accessibility in Tanzania as well as lack of trained personnel to recognize the condition may be the reason why the patient was diagnosed much later in life. For this reason, there is also a lack of statistics that detail the incidence and prevalence of DS in Tanzania.
Primary prevention of the traumatic spinal cord injuries is an important public health measure and a heightened emphasis should be placed to prevent such injuries in highrisk populations including DS patients. Late presentation and loss to follow-up are common challenges we face in our patient population, thus highlighting the importance of creating public health awareness and implementing primary prevention measures [15].

\section{Conclusion}

In summary, AAI in patients with DS is recognized, and a high index of suspicion should be maintained by healthcare personnel who come across patients with DS presenting with cervical spine complaints such as neck pain, progressive pyramidal signs and symptoms, or peripheral neurological deficits. Counselling caregivers of DS patients about the associated risk of neck injury is an important public health intervention.

\section{Compliance with ethical standards}

Conflict of interest The authors declare that they have no conflict of interest.

\section{References}

1. Centers for Disease Control and Prevention. Data and Statistics: occurrence of Down Syndrome in the United States. https://www. cdc.gov/ncbddd/birthdefects/downsyndrome/data.html. Accessed 26 July 2018.

2. McKelvey KD, Fowler TW, Akel NS, Kelsay JS, Gaddy D, Wenger GR, et al. Low bone turnover and low bone density in cohort of adults with Down syndrome. Osteoporos Int. 2013;4:1333-888.

3. Hawli Y, Nasrallah M, Fuleihan GE. Endocrine and musculoskeletal abnormalities in patients with Down syndrome. Nat Rev Endocrinol. 2009;6:327-34.

4. Galli M, Rigoldi C, Brunner R, Virji-Babul N, Giorgio A. Joint stiffness and gait pattern evaluation in children with Down syndrome. Gait Posture. 2008;3:502-6.

5. Lott IT. Neurological phenotypes for Down syndrome across the life span. Prog. Brain Res. 2012;197:101-21.

6. Roy M, Baxter M, Roy A. Atlantoaxial instability in Down syndrome-guidelines for screening and detection. J R Soc Med. 1990;7:433-5.

7. El-Khouri M, Mourão MA, Tobo A, Battistella LR, Herrero CF, Riberto M. Prevalence of atlanto-occipital and atlantoaxial instability in adults with Down syndrome. World Neurosurg. 2014;1-2:215-8.

8. Myśliwiec A, Posłuszny A, Saulicz E, Doroniewicz I, Linek P, Wolny T, et al. Atlanto-axial instability in people with Down's syndrome and its impact on the ability to perform sports activities - a review. J Hum Kinet. 2015;1:17-24.

9. Iwase S, Inukai Y, Nishimura N, Sato M, Sugenoya J. Hemifacial hyperhidrosis associated with ipsilateral/contralateral cervical disc herniation myelopathy. Functional considerations on how 
compression pattern determines the laterality. Funct Neurol. 2014;1:67-73.

10. Kirshblum SC, Waring W, Biering-Sorensen F, Burns SP, Johansen M, Schmidt-Read M, et al. Reference for the 2011 revision of the international standards for neurological classification of spinal cord injury. J Spinal Cord Med. 2011;1:547-54.

11. Pueschel SM, Herndon JH, Gelch MN, Senft KE, Scola FH, Goldberg MJ. Symptomatic atlantoaxial subluxation in persons with Down syndrome. J Pediatr Orthop. 1986;6:682-8.

12. Yang SY, Boniello AJ, Poorman CE, Chang AL, Wang S, Passias PG. A review of the diagnosis and treatment of atlantoaxial dislocations. Glob Spine J. 2014;3:197-210.
13. Stanifer JW, Patel UD, Karia F, Thielman N, Maro V, Shimbi D, et al. The determinants of traditional medicine use in northern Tanzania: a mixed-methods study. PLoS One. 2015;10:e122638.

14. The World Bank. Physicians (per 1, 000 people), 2014 [Internet]. https://data.worldbank.org/indicator/SH.MED.PHYS.ZS?loca tions $=$ TZ\&name_desc $=$ false. Accessed 2 Nov 2018.

15. Dekker MC, Urassa SJ, Howlett WP. Neurological letter from Kilimanjaro. Pract Neurol. 2017;5:412-6.

16. Medical Research Council. Memorandum Number 45. Aids to the examination of the peripheral nervous system. London: HM Stationery Office; 1976. 